\title{
Online Products Fake Reviews Detection System Using Machine Learning
}

\author{
Deepika Vachane', G.D. Upadhye ${ }^{2}$ \\ 1 JSPM's Rajarshi Shahu College of Engg. Pune, India \\ 2 JSPM's Rajarshi Shahu College of Engg. Pune, India
}

Article History: Received: 10 November 2020; Revised: 12 January 2021; Accepted: 27 January 2021; Published online: 05 April 2021

\begin{abstract}
Nowadays online analyses gotten single indispensable components clients web based shop. Associations plus people utilize data near purchase correct items then settle on business choices.affect spammers otherwise unscrupulous agents make bogus surveys also elevate items near rivalries. To handle this issue, examines have been directed to define successful approaches to recognize the spam surveys. Different spam recognition strategies have been presented in which a large portion of them separates significant highlights from the content or utilized AI proceduresIn this paper, named spam recognition framework, which uses spam highlights for showing review enlightening lists as heterogeneous information structures to configuration spam ID strategy into a gathering of issue. Utilizing the criticalness, us to acquire great results with respect to various measurements on survey informational indexes. The commitment effort remains after people search enquiry show wholly n-no of things similarly as recommendation of the thing.
\end{abstract}

Keywords: Fake Review, Machine Learning, Netspam Algorithm, Sentiment Analysis Algorithm, Semantic Analysis Algorithm, Social Media, Social Network, Spammer, Spam Review

\section{Introduction}

The world is seeing increasingly more support in present day electronic trade, where online reviews is assuming an indispensable job. Customer presently participate in perusing reviews on items and stores when they are settling on choices on what to purchase or where to get it. Spam analysts took advantage of this lucky break to compose pernicious reviews to ruin fair stores or utilize counterfeit surveys to hoodwink customers on low quality items. This is regularly viewed as spam reviews. These spam reviews had represented a genuine danger to web-based business, with people, organizations, coordinates and associations loosing colossal whole of fortune all the while. Customers suppositions assume an indispensable job in purchasing choices. Nowadays the greater part of the customer posts their feeling for items on online journals, web-based business locales, reviews destinations and person to person communication destinations. The above data are devoured by business or corporate associations, as they are energetically keen on examining the customer sees about their items, administrations and backing. As individuals purchase items subsequent to perusing the surveys, the sort of reviews that an item draws in is of worry to the dealers. This implies a positive survey on item would acquire deals and a negative one would diminish them.

\section{Related Work}

In [1] paper, Spam crusades seen in well-known item audit sites (e.g., amazon. com) pulled in growing consideration starting equally trade also the scholarly community, gathering wired banners is employed to cooperatively make tricky audits for some objective goad cuts. The objective is to control seen notorieties of the objectives for their eventual benefits. they discover disjoint sets connected straight and concrete perfect the social nearness betrayer e.g., appraising related merchandises and saying related thoughts in tiny period.

In [2] paper, Online thing studies have become a huge wellspring of customer sentiments. Due to profit or reputation, cheats have been creating dubious or counter-feint re-points of view to progress or possibly to minimize some target things or organizations. Such imposters are called review spammers. A chart spread means for finding junk reviews accessible. innovative estimate scheme established directed knowledge defined fact with the hard tricky of estimation deprived of milled reality facts, sorts analyses built on dissimilar topographies as of topographies charity in finding junk reviews.

In [3] paper, Online surveys on items and administrations can be helpful for customers, however they should be shielded from control. Up until now, most examinations have zero in on dissecting on the web surveys from a solitary facilitating webpage. How might one influence data from numerous audit facilitating destinations? This is the vital inquiry in our work. Accordingly, build up a precise philosophy to union, look at, and assess audits from various facilitating destinations. Zero in on lodging surveys and utilize more than 15million audits from more than 3.5 million clients spreading over three noticeable travel destinations. 
In [4] paper, Users progressively depend on publicly supported data, for example, rises on Yelp and Amazon, and preferred post sand promotions on Facebook. This has lento market for dark cap advancement strategies by means of phony (e.g., Sybil) and traded off records, and intrigue organizations. Existing ways to deal with distinguish such conduct re-lies generally on regulated (or semi-managed) learning over known (or hypothecsized) assaults. They can't distinguish assaults missed by the administrator while labeling, or when the assailant changes methodology.

In [5] paper, Online surveys have become an inexorably significant asset for dynamic and item planning. Yet, audits frameworks are frequently focused by sentiment spamming. Although genuine audit identification has been read by researchers for quite a long time utilizing managed learning, ground reality of huge scope datasets is as yet inaccessible and the vast majority of existing methodologies of regulated learning depend on pseudo phony surveys as opposed to genuine phony surveys.

In [6] paper, Online audits are rapidly getting one of the main wellsprings of data for shoppers on different items and administrations. With their expanded significance, there exists an expanded open door for spammers or deceptive entrepreneurs to make bogus audits so as to misleadingly advance their products and enterprises or slight those of their rivals. Because of this developing issue, there have been numerous examinations on the best methods of recognizing survey spam utilizing different AI calculations. One ongoing idea in the majority of these investigations is the change of surveys to word vectors, which can possibly bring about countless highlights.

In [7] paper, it giving a beneficial and convincing procedure to perceive analysis spammers melding public associations subject to dual notions individuals will undoubtedly ruminate analyses since folks related per trustworthy, overview spammers remain fewer disposed keep up colossal bond link per run of mill customers. The duties of this paper are two-cover:

(1) Clarify how social associations can be joined into review rating desire and propose a trust based rating figure model using closeness as trust weight.

(2) Design a trust-careful distinguishing proof model reliant on rating change which iteratively calculates customer express as a rule constancy scores as the marker for spam city.

In [8] paper, to identify counterfeit audits for an item by utilizing the content and rating property from a survey. To put it plainly, suggested framework (ICF++) quantify genuineness estimation survey, trustiness estimation analysts plus unwavering quality estimation item. Part-Of-Speech (POS) despoiler stanfordcorenlp reads script then decide amount of dialog separately symbol. method made tab contractions, for English, despoiler ensued contractions Penn Treebank ordinary.

In [9] paper, Online Social Networks (OSNs), which catches the structure and dynamics of individual toindividual and individual to innovation association, is being utilized for different purposes, for example, business, schooling, selling, clinical, entertainment. This innovation additionally opens the entryway for unlawful exercises. Identifying oddities, in this new viewpoint of public activity that explains and mirrors the disconnected connections, is a significant factor as they could be an indication of a significant not issue or conveying valuable data for the analyzer.

In [10] paper, they propose another all-encompassing methodology considered SpEagle that uses signs from all metadata (text, timestamp, and rating) just as social information (organization), and saddle them by and large under a bound together framework to spot suspicious clients and surveys, just as items focused by spam. SpEagle utilizes an audit network-based order task which acknowledges earlier information on the class dissemination of the hubs, assessed from metadata. Positive focuses are: It empowers consistent incorporation of named information when accessible. It is very efficient.

In [11] paper, an innovative framework, titled Net Spam use spam feature aimed at showing analysis datasets as mixed material associations near configuration spam area technique hooked on plan issue associations. Using the significance of spam features secure well again achieves terms of Special estimations test genuine overview datasets from Yelp and Amazon destinations. inspection that maped load using meiosis ideac potent identifying junk reviews and leads to a good performance.

In [12] paper, The Convolution Neural Network(CNN) and Particle Swarm Optimization (PSO), those two methodologies use for acknowledgment of the disengaged manually written digit. Altered PSO is utilized to lessen the general calculation season of the proposed framework. focused on altered PSO with CNN based model for perceiving transcribed digits. Preparing of the $\mathrm{CNN}$ model is an exceptionally troublesome errand and this 
sets aside a long effort for calculation. Furthermore, equipment turns into a major issue in preparing these models.

In [13] paper, Mango evaluated now sorts like Green Mango, Yellow Mango and Red Mango depend AI technique. framework studies RGB esteems scope then state mango. Following investigation is utilized to get great likelihood. assists with preparing framework to distinguish suitable development of mangoes. This exploration is led on two AI strategy for example Innocent Byes and SVM (Support Vector Machine).

In [14] paper, Design characterization, transcribed digit order is a difficult issue. Manually written digits appear to be changed because of composing styles and sizes.

\section{Findings}

Another proposed system comprises in speaking to a bunch of surveys information gave as HIN plus understanding difficult junk finding matter HIN ranking. Specifically, to show their perspectives data record by way of a HIN where the studies connected over several kinds of hubs, (aimed at case, usefulness plus customers). At that point premium intention consumed figure the importance (or weight) of each capacity. These loads are utilized to ascertain the latest audit marks utilizing managed and unaided strategies. graceful observations, defining dual angles on behalf of climaxes (study customer and social semantic), classified acmes audit conduct take extra loads vintage well finishing proceeding recognizing junk surveys equally directed plus unaided approaches.

\subsection{Architecture:}

The Fig.1 shows the proposed system architecture.

1) NetSpam method unique organization built methodology copies rise net-works as mixed data organizations.

2) Additional allowance tactic expected junk acmes stays planned near agree the relative worth each element and shows how viable every one of highlights are in recognizing spams from ordinary surveys.

3) NetSpam system improves the exactness against the cutting edge in purposes of time unpredictability, which very be contingent extent acmes castoff distinguish junk audit.

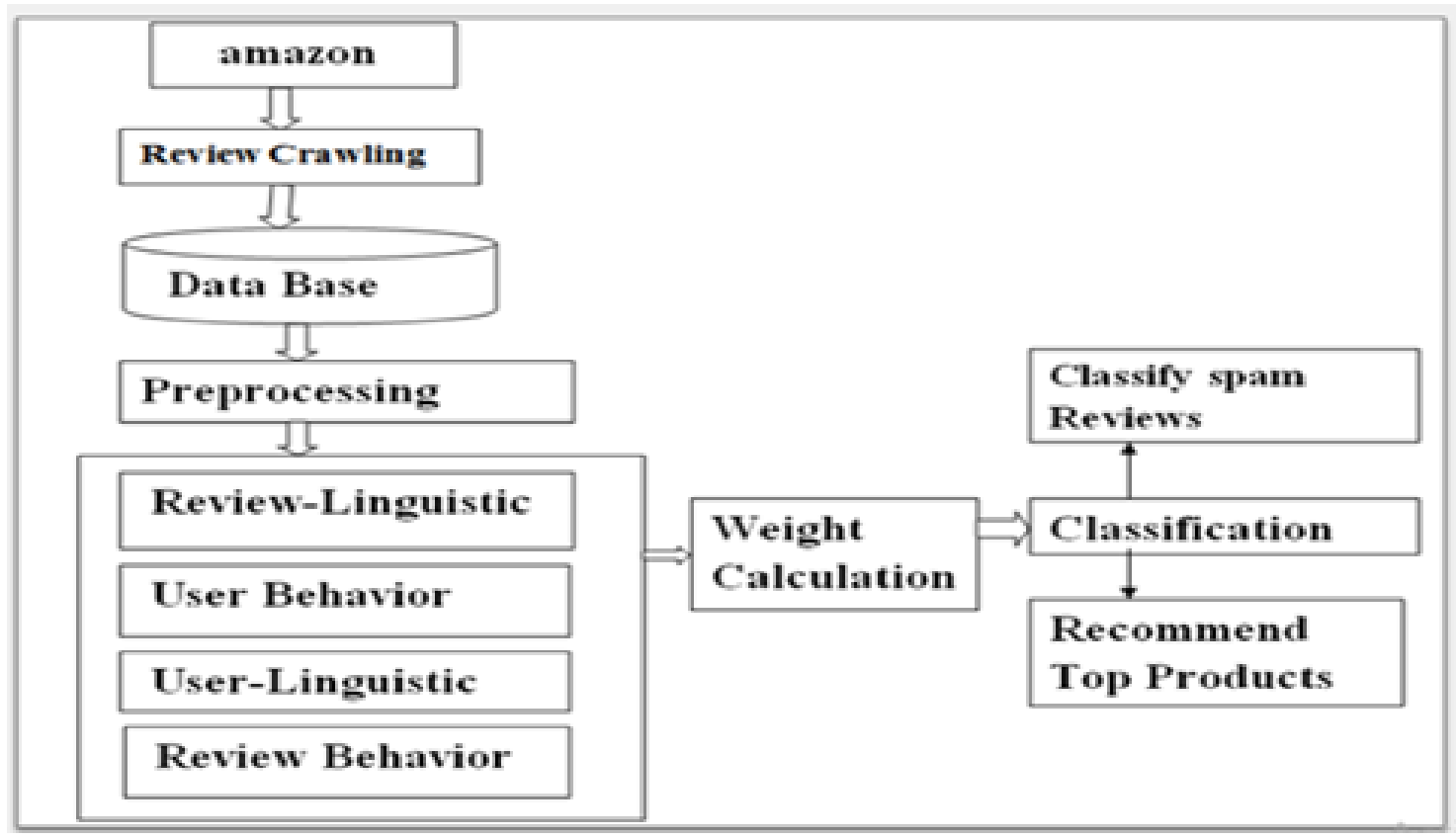

Figure 1. Proposed System Architecture 


\subsection{Algorithms}

\section{Sentiment Analysis Algorithm:}

Input: Text File (comment or review) T, The sentiment lexicon L. Output: $\mathrm{Smt}=\{\mathrm{P}, \mathrm{Ng}$ and $\}$ and strength $\mathrm{S}$ where $\mathrm{P}$ :

Positive, Ng: Negative, N: Neutral

Initialization: SumPos $=$ SumNeg $=0$, where,

Sum Pos: accumulates the polarity of positive tokens ti smt in T, Sum Neg: accumulates the polarity of negative tokens ti smt in T, Begin

1.For each $\mathrm{ti} \in \mathrm{T}$ do

2.Search for ti in $\mathrm{L}$

3. If ti $\in$ Pos-list then

4. SumPos $\leftarrow$ SumPos $+\mathrm{ti}^{-}-\mathrm{smt}$

5. Else ifti $\in$ Pos-list then

6. Sum Neg $\leftarrow$ Sum Neg + ti - smt

7. End If

8. End For

9. If SumPos $>\mid$ Sum Neg| then

10. $\mathrm{Smt}=\mathrm{P}$

11. $\mathrm{S}=$ SumPos/ (Sum Pos+Sum Neg)

12. Else If Sum Pos $<\mid$ Sum Neg $\mid$ then

13. $\mathrm{Smt}=\mathrm{Ng}$

14. $\mathrm{S}=\mathrm{Sum} \mathrm{Neg} /(\mathrm{Sum} \mathrm{Pos}+\mathrm{Sum} \mathrm{Neg})$

15. Else

16. $\mathrm{Smt}=\mathrm{N}$

17. $\mathrm{S}=$ SumPos/ (Sum Pos+Sum Neg)

18. End If End

\section{Latent Semantic Analysis Algorithm}

1)Step 1: Documents should be prepared in the following way:

- Exclude trivial words as well as low-frequency terms.

- Conflate terms with techniques like stemming or lemmatization.

2) Step 2: A term-frequency matrix (A) must be created that includes the occurrences of each term in each document.

3) Step 3: Singular Value Decomposition (SVD):

- Extract least-square principal components for two sets of variables: set of terms and set of documents.

- SVD products include the term eigenvectors $\mathrm{U}$, the document eigenvectors $\mathrm{V}$, and the diagonal matrix of singular values $P$.

4) Step 4: From these, factor loadings can be produced for terms UP and documents VP

\section{Netspam Algorithm}

following algorithm is taken from references no [11];

- Input: review-dataset, spam-feature-list, pre-labeled reviews

- Output: features importance (W), spamicity probability (Pr) - Process:

- Step 1: u, v: review, yu: spamicity probability of review u

- Step 2: $\mathrm{f}(\mathrm{xlu})$ : initial probability of review u being spam

- Step 3: Pl: metapath based on feature 1, L: features number

- Step 4: n: number of reviews connected to a review

- Step 5: mPl u: the level of spam certainty

- Step 6: mPl u, v: the metapath value

- Step 7: Prior Knowledge 
- Step 8: if semi-supervised mode

- Step 9: if $u \in$ pre-labeled-reviews

- Step 10: yu = label(u)

- Step 11: else

- Step 12: yu =0

- Step 13: else unsupervised mode

- Step 14: yu = 1 LPL l=1 f(xlu)

- Step 15: Network Schema Definition

- Step 16: schema = defining schema based on spam

feature-list

- Step 17: Metapath Definition and Creation

- Step 18: for pl $\in$ schema

- Step 19: for $u, v \in$ review-dataset

- Step 20: $\mathrm{mpl} \mathrm{u}=|\mathrm{s} \times \mathrm{f}(\mathrm{xlu})| \mathrm{s}$

- Step 21: $\mathrm{mpl} \mathrm{v}=|\mathrm{s} \times \mathrm{f}(\mathrm{xlv})| \mathrm{s}$

- Step 22: if $\mathrm{mpl} \mathrm{u}=\mathrm{mpl} \mathrm{v}$

- Step 23: mppl u, v = mpl u

- Step 24: else

- Step 25: mppl u, v =0

- Step 26: Classification - Weight Calculation

- Step 27: for $\mathrm{pl} \in$ schemes

- Step 28: $\mathrm{Wpl}=\mathrm{Pn} \mathrm{r}=1 \mathrm{Pn} \mathrm{s}=1 \mathrm{mp}$ pl r,s $\times$ yr $\times$ ysP n r=1Pn s=1 mp pl r,s

- Step 29: Classification - Labeling

- Step 30: for $u, v \in$ review-dataset

- Step 31: Pru,v = 1-QL pl=1 1-mppl u,v $\times$ Wpl

- Step 32: Pru = avg (Pru,1, Pru, 2..., Pru, n)

\section{Result and Discussion}

\section{Dataset:}

We used amazon product reviews dataset 800 reviews uses in research. compared straightforward and dishonest helpful and harmful analyses on behalf of products establish happening amazon application.

\section{Result and Analysis:}

The section shows overall accuracy of Existing Algorithm and proposed algorithm. So, this works gives better results compare to existing method. 


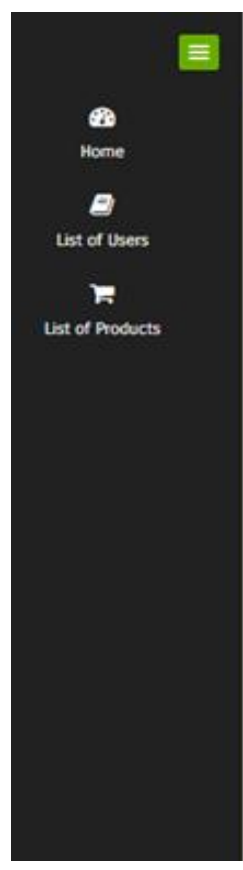

\section{NetSpam Framework}

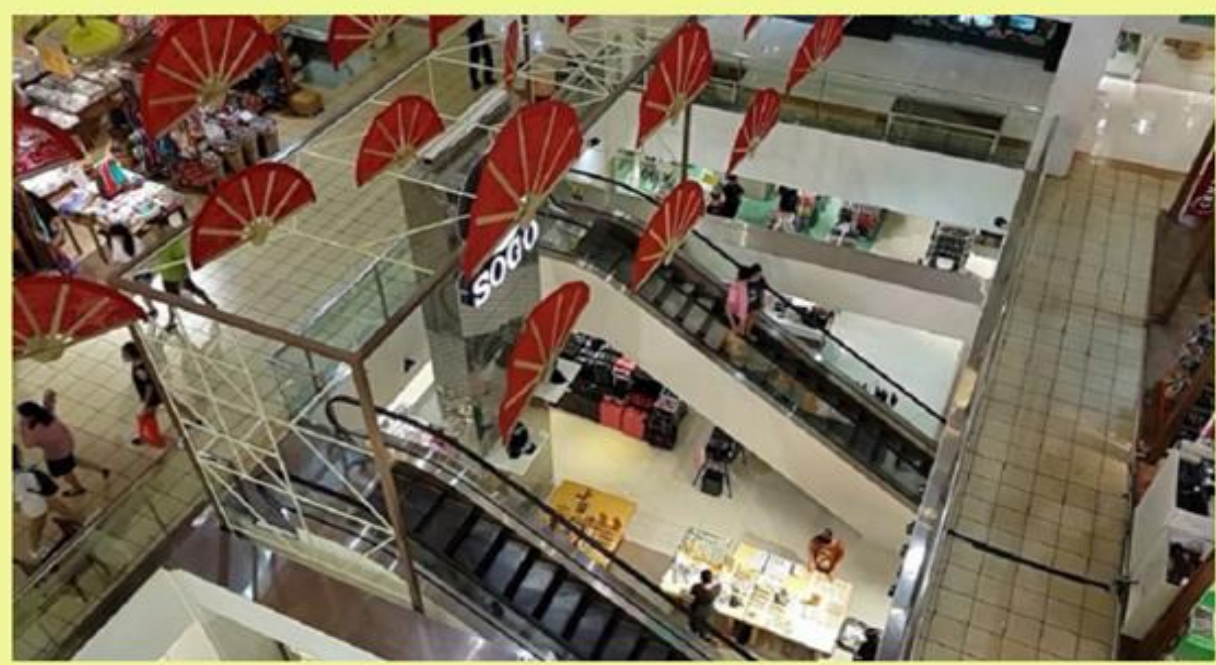

Figure 1. Home Page

List of Products

\begin{tabular}{|c|c|c|c|c|c|c|c|}
\hline Id & Image & Item ld & Item Name & Price & Rating & Category & Reviews \\
\hline 1 & & B075625NB & OnePlus 5T (Vioright Black 698 RAM + 64GB memony) & 32999.0 & 45 & Mobile & Extrat \\
\hline 2 & & BOIFMS732 & Lava 152 (Black, 468) & 3599.0 & 25 & Mobile & Etrast \\
\hline 3 & & BOTSHBHSKV & Vino V9 (19:9 Fullvew Display, Pearl Black) with Offers & 2399000 & 45 & Nobils & Extrat: \\
\hline 4 & & BOTHWVTHPH & Noto G5s Plus (Lunar Grey, 64GB) & 10999.0 & 3.8 & Noble & Ettats \\
\hline
\end{tabular}

Figure 1.2 List of Products 
Online Products Fake Reviews Detection System using Machine Learning

\begin{tabular}{|c|c|c|c|c|c|}
\hline Customer Name & Title & Rating & Verified Purchase & Review Date & Content \\
\hline Ranjeet & Two Stars & 2.0 & true & lewed in india on 5 July 2018 & Button damage nikla \\
\hline Govind pandey & Four Stars & 40 & true & iewed in India on 27 April 2018 & Product good but size small .... \\
\hline Amazon Customer & Smaller than expected & 1.0 & true & lewed in India on 10 September 2017 & The pant is too small \\
\hline Ranjeet & Two Stars & 2.0 & true & iewed in india on 5 July 2018 & Button damage nikla \\
\hline Govind pandey & Four Stars & 4.0 & true & lewed in India on 27 April 2018 & Product good but size smail .... \\
\hline Amazon Customer & Smaller than expected & 10 & true & lewed in India on 10 September 2017 & The pant is too small \\
\hline Ranjeet & Two Stars & 2.0 & true & iewed in India on 5 July 2018 & Button damage nikla \\
\hline Govind pandey & Four Stars & 4.0 & true & lewed in India on 27 April 2018 & Product good but size small .... \\
\hline Amazon Customer & Smaller than expected & 10 & true & lewed in India on 10 September 2017 & The pant is too small \\
\hline Ranjeet & Two Stars & 2.0 & true & lewed in India on 5 July 2018 & Button damage nikla \\
\hline Govind pandey & Four Stars & 4.0 & true & lewed in India on 27 April 2018 & Product good but size small .... \\
\hline Amazon Customer & Smaller than expected & 1.0 & true & lewed in India on 10 September 2017 & The pant is too small \\
\hline
\end{tabular}

Figure 1.3 List of Reviews

\begin{tabular}{|c|c|c|c|c|}
\hline \multicolumn{5}{|c|}{ Sentiment Analysis on List of Reviews } \\
\hline Review ID & Customer Name & Content & Sentiment Score & Sentiment Result \\
\hline RIZQBUP910F6LO & Ranjeet & Button damage nikla & 0.7 & Neutral \\
\hline R2IIXCGSIM93Q & Govind pandey & Product good but size small .... & 10 & Positive \\
\hline RCDMLMgEUBIN1 & Amazon Customer & The pant is too small. & 0.0 & Negative \\
\hline RIZQBUP91DF6LO & Ranjeet & Button damage nikla & 0.7 & Neutral. \\
\hline R2\|IXCGSIM93Q & Govind pandey & Product good but size small .... & 10 & Positive \\
\hline RCDMLMgEU8IN1 & Amazon Customer & The pant is too small. & 0.0 & Negative \\
\hline RIZQQUP91DF6LO & Ranjeet & Button damage nikla & 0.7 & Neutral \\
\hline R2IIXCGSIM93Q & Govind pandey & Product good but size small .... & 10 & Positive \\
\hline RCDMLM9EU81N1 & Amazon Customer & The pant is too small. & 0.0 & Negative \\
\hline RIZQBUP910F6LO & Ranjeet & Button damagre nikla & 0.7 & Neutral \\
\hline R2IIXCGSIM930 & Govind pander & Product good but size small ... & 10 & Positive \\
\hline
\end{tabular}

Figure1.4 Sentiment Analysis 


\begin{tabular}{|c|c|c|c|}
\hline \multicolumn{4}{|c|}{ Semantic Analysis on List of Reviews } \\
\hline Review ID & Customer Name & Content & Semantic Score \\
\hline RIZQQBUP910F6LO & Ranjeet & Button damage nikla & 12.0 \\
\hline R2IIXXCGSIM93Q & Govind pandey & Product good but size small .... & 12.0 \\
\hline RCDMLMgEUBIJ1 & Amazon Customer & The pant is too small & 12.0 \\
\hline RIZQQBUP91DF6LO & Ranjeet & Button damage nikla & 12.0 \\
\hline R2IIXCGSIM93Q & Govind pandey & Product good but size small .... & 12.0 \\
\hline RCDMLMgEUBiN1 & Amazon Customer & The pant is too small. & 12.0 \\
\hline RIZQQBUPG1DF6LO & Ranjeet & Button damage nikla & 12.0 \\
\hline R2IIXCGSIM93Q & Govind pandey & Product good but size small .... & 12.0 \\
\hline RCDMLM9EUBUI1 & Amazon Customer & The pant is too small. & 12.0 \\
\hline RIZQBUP910F6LO & Ranjeet & Button damage nikla & 12.0 \\
\hline R2IIIXCGSIM930 & Govind pandey & Product good but size small .... & 12.0 \\
\hline
\end{tabular}

Figure 1.5 Semantic Analysis

\begin{tabular}{|c|c|c|c|}
\hline \multicolumn{4}{|c|}{ Spam Detection on List of Reviews } \\
\hline Renier ID & $\begin{array}{l}\text { Costomer } \\
\text { Name }\end{array}$ & Content & Olassity \\
\hline RHFTYHOWNLLB & S. Panot: & 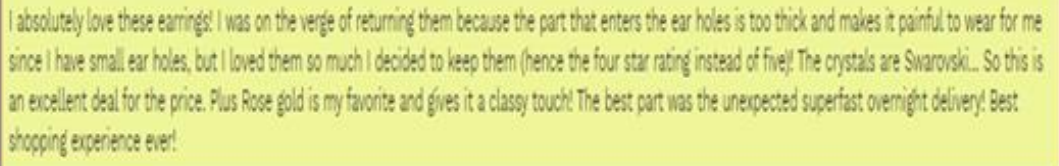 & Scam \\
\hline Ronougasiong & suthy & 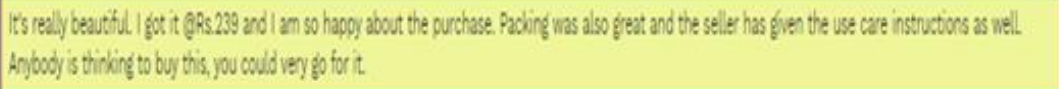 & $\begin{array}{l}\text { Non- } \\
\text { spean }\end{array}$ \\
\hline RELALLLZVM & Shrobtha & 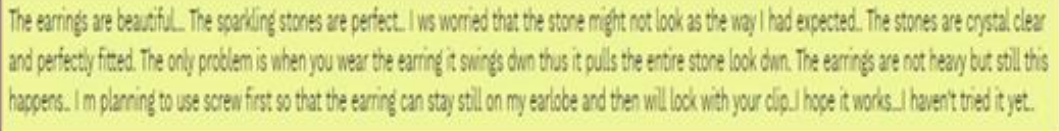 & Ssean \\
\hline REWWGKOSS85 & $1 / a$ & 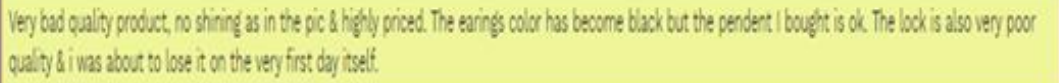 & Seam \\
\hline RSGTKENGAPZ & arker anasti & 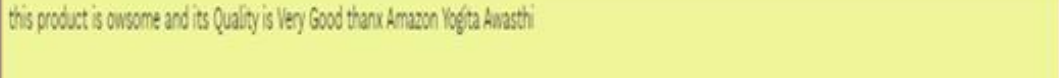 & $\begin{array}{l}\text { Nor- } \\
\text { speem }\end{array}$ \\
\hline
\end{tabular}

Figure 1.6 Spam Detection 


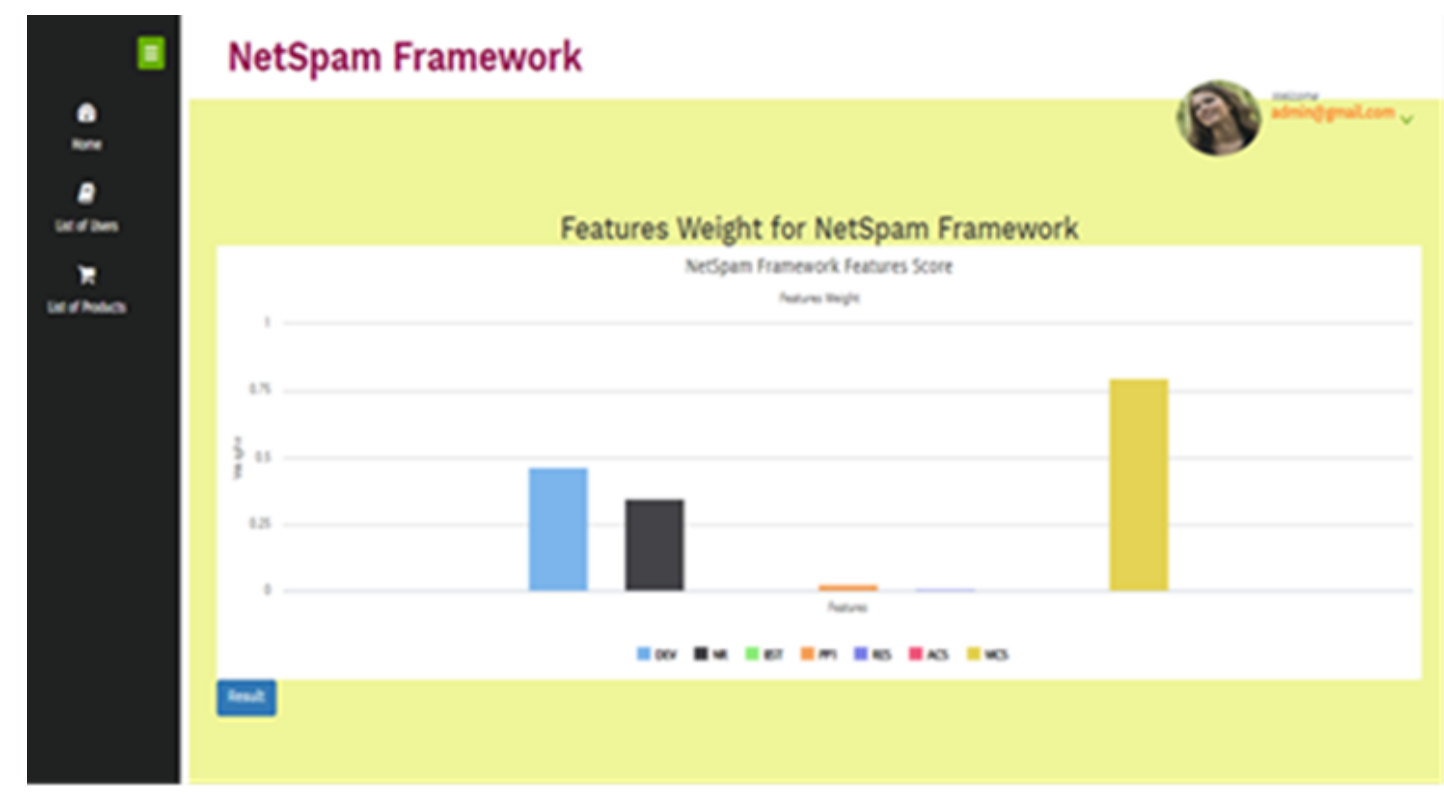

Figure 1.7. Features Weight for Netspam

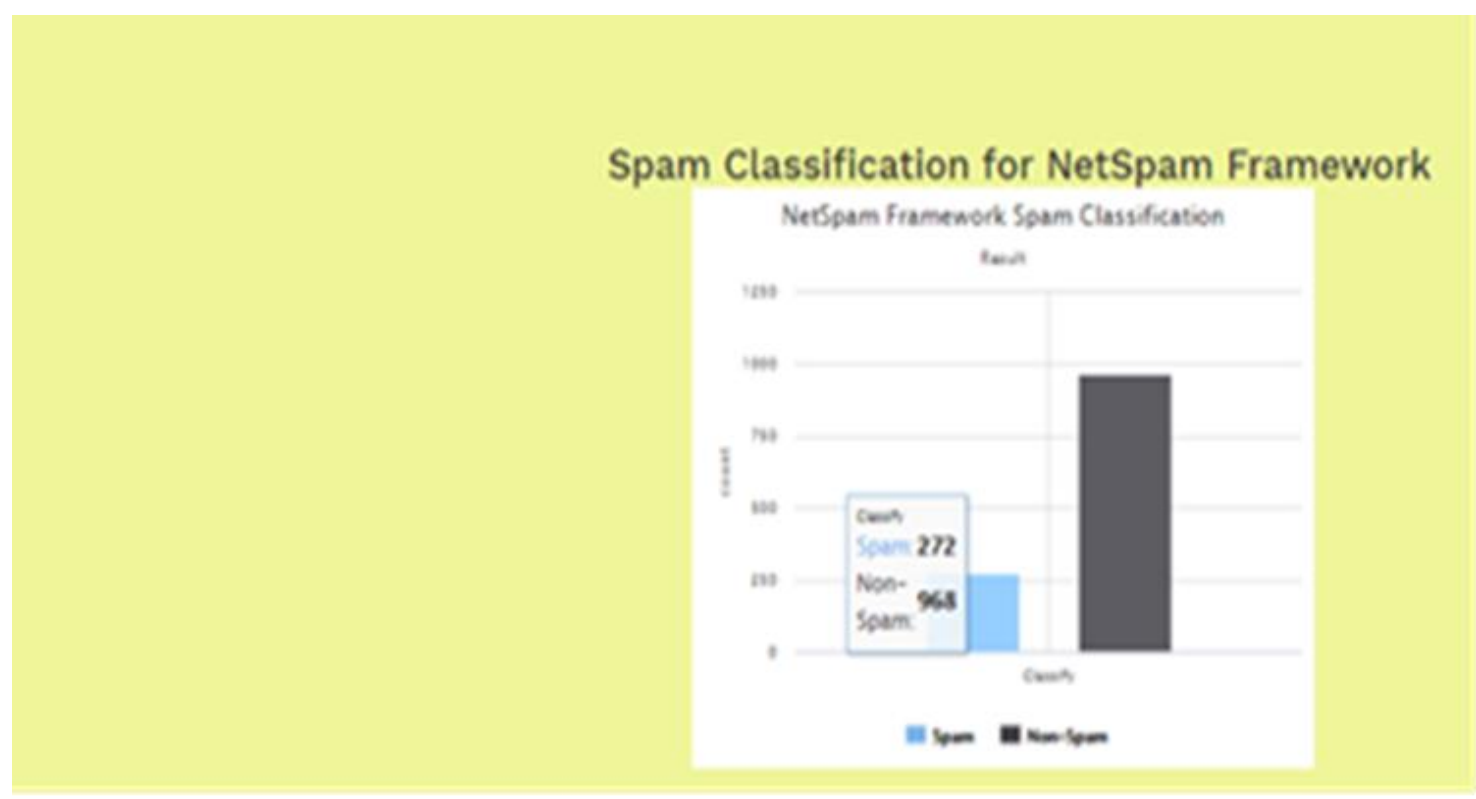

Figure 1.8. Spam Classification 


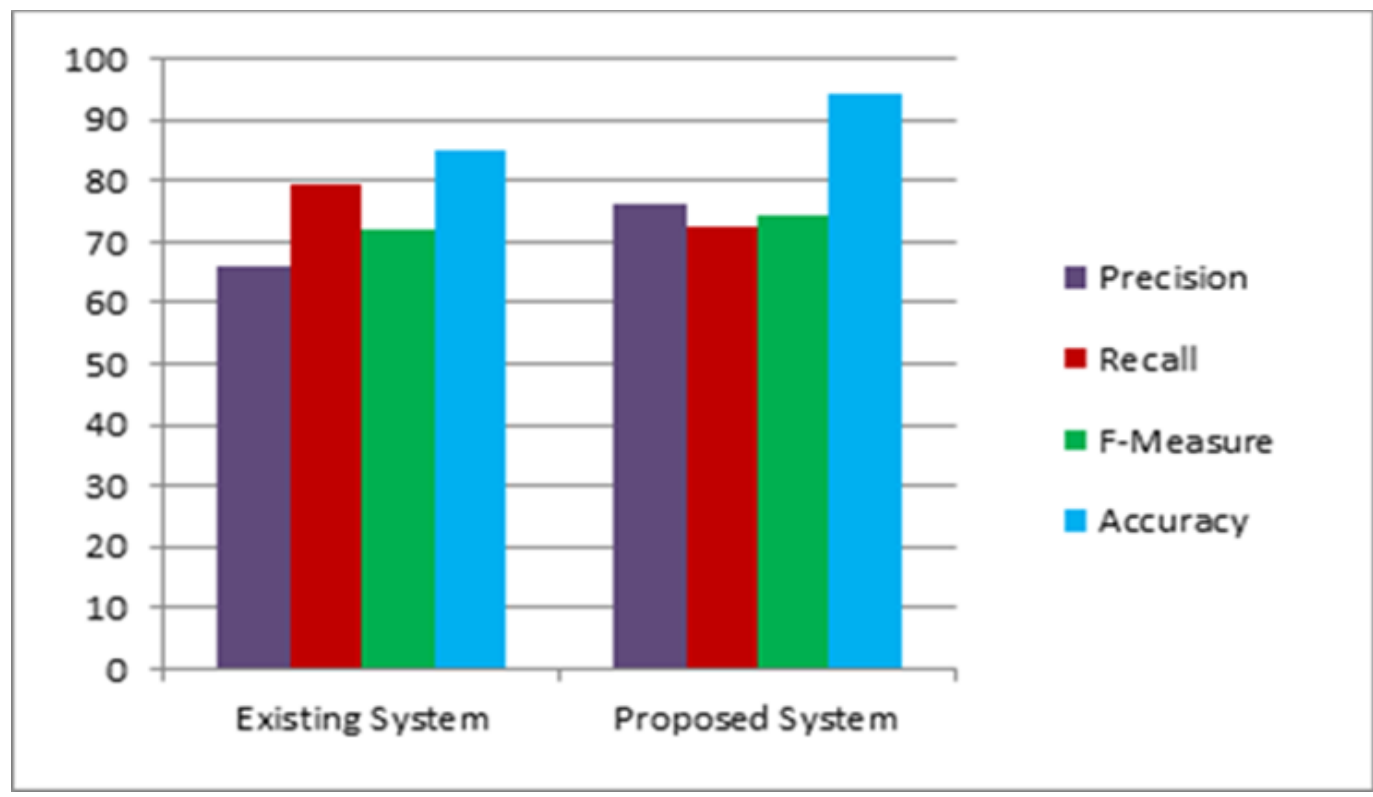

Figure 1.9. Comparison Graph

\begin{tabular}{|l|l|l|}
\hline Sr. No. & Existing System & Proposed System \\
\hline Algorithm & Naive Bayes & Decision Tree \\
\hline Precision & $60.2 \%$ & $65.4 \%$ \\
\hline Recall & $85.5 \%$ & $89.7 \%$ \\
\hline Accuracy & Accuracy: $85.14 \%$ & Accuracy: $92.06 \%$ \\
\hline
\end{tabular}

Figure 1.10 Comparison Table

\section{Conclusion}

In this proposed system investigation benevolences innovative spam finding structure certain NetSpam framework for product reviews based on Sentiment analysis (SA) and latent semantic analysis (LSA). This paper has used SA and LSA with netspam algorithm for spam detection. Additionally, NetSpam can end furthermore, it profits superior finishing highpoint extension method, plus executes higher all former mechanism, per even handed retiring numeral acmes. Tallying rouse branding prime classifications aimed at acmes sensitivities reveal analyses behaviour ranking. LSA is used in the proposed system to reduce similar comments and try to improve spam detection accuracy. The outcomes about additionally that developing individual observations, reasonable towards the semi-supervised skill, take nope observable sway happening pivotal the vast majority of the subjective climaxes, equally by way of in several datasets. 


\section{References}

1. Ch. Xu and J. Zhang," Combating product review spam campaigns via multiple heterogeneous pairwise features", In SIAM International Conference on Data Mining, 2014.

2. G. Fei, A. Mukherjee, B. Liu, M. Hsu, M. Castellanos, and R. Ghosh, "Exploiting bustiness in reviews for review spammer detection", In ICWSM, 2013.

3. A. j. Minnich, N. Chavoshi, A. Mueen, S. Luan, and M. Faloutsos, "True view: Harnessing the power of multiple review sites", In ACM WWW, 2015.

4. B. Viswanath, M. Ahmad Bashir, M. Crovella, S. Guah, K. P. Gummadi, B. Krishnamurthy, and A. Mislove, "Towards detecting anomalous user behavior in online social networks", In USENIX, 2014.

5. H. Li, Z. Chen, B. Liu, X. Wei, and J. Shao," Spotting fake reviews via collective PU learning”, In ICDM, 2014.

6. M. Crawford, T. M. Khoshgoftaar, and J. D. Prusa," Reducing Feature Set Explosion to Faciliate Real-World Review Spam Detection", In Proceeding of 29th International Florida Artificial Intelligence Research Society Conference, 2016.

7. H. Xue, F. Li, H. Seo, and R. Pluretti," Trust-Aware Review Spam Detection", IEEE Trustcom/ISPA.,2015.

8. E. D. Wahyuni, A. Djunaidy," Fake Review Detection from a Product Review Using Modified Method of Iterative Computation Framework", In Proceeding MATEC Web of Conferences, 2016.

9. R. Hassanzadeh," Anomaly Detection in Online Social Networks: Using Datamining Techniques and Fuzzy Logic", Queensland University of Technology, Nov, 2014.

10. R. Shebuti, L. Akoglu," Collective opinion spam detection: bridging review networks and metadata", In ACM KDD, 2015.

11. Saeedreza Shehnepoor, Mostafa Salehi*, Reza Farahbakhsh, Noel Crespi," Netspam: a networkbased spam detection framework for reviews in online social media", IEEE conference paper, 2017.

12. G.D. Upadhye, P. Barhate, "Classifying Handwritten Digit Recognition Using CNN and PSO", IJRTE, ISSN: 2277-3878, Volume-8 Issue-2, July 2019.

13. G.D. Upadhye, D. Pise, "Grading of Harvested Mangoes Quality and Maturity Based on Machine Learning Techniques", IEEE International conference on smart city and Emerging Technology,2018.

14. Upadhye G.D., Kulkarni U.V. (2020)," Pattern Classification of Handwritten Kannada Digits Using Customized CNN". In: Reddy V., Prasad V., Wang J., Reddy K. (eds) Soft Computing and Signal Processing. ICSCSP 2019. Advances in Intelligent Systems and Computing, vol 1118. Springer, Singapore. 